P. Jichlinski · G. Wagnières $\cdot$ M. Forrer $\cdot$ J. Mizeret

L. Guillou $\cdot$ M. Oswald · F. Schmidlin $\cdot$ P. Graber

H. Van den Bergh $\cdot$ H.-J. Leisinger

\title{
Clinical assessment of fluorescence cystoscopy during transurethral bladder resection in superficial bladder cancer
}

\begin{abstract}
The prognosis of superficial bladder cancer in terms of recurrence and disease progression is related to bladder tumor multiplicity and the presence of concomitant "plane" tumors such as high-grade dysplasia and carcinoma in situ. This study in 33 patients aimed to demonstrate the role of fluorescence cystoscopy in transurethral resection of superficial bladder cancer. The method is based on the detection of protoporphyrin-IX-induced fluorescence in urothelial cancer cells by topical administration of 5-aminolevulinic acid. The sensitivity and the specificity of this procedure on apparently normal mucosa in superficial bladder cancer are estimated to be $82.9 \%$ and $81.3 \%$, respectively. Thus, fluorescence cytoscopy is a simple and reliable method for mapping the bladder mucosa, especially in the case of multifocal bladder disease, and it facilitates the screening of occult dysplasia.
\end{abstract}

Key words Carcinoma - Transitional cell - Cystoscopy Fluorescence $\cdot 5$-Aminolevulinic acid $\cdot$ Protoporphyrin IX

\section{Introduction}

In superficial bladder transitional cell carcinoma, tumor multiplicity and concomitant urothelial atypia are indicators of a poor disease prognosis [1,2]. High-grade dyspla-

P. Jichlinski (夰) - M. Oswald · H.-J. Leisinger Department of Urology, University Hospital, CHUV, CH-1011 Lausanne, Switzerland

$\mathrm{G}$. Wagnières $\cdot$ M. Forrer $\cdot J$. Mizeret $\cdot H$. Var den Bergh

Laboratory of Environmental Engineering,

Swiss Federal Institute of Technology (EPFL),

CH-1015 Lausanne, Switzerland

L. Guillou

Institute of Pathology, University Hospital, CHUV, CH-1011 Lausanne, Switzerland

F. Schmidlin · P. Graber

Department of Urology, University Hospital,

HCUG, CH-1205 Geneva, Switzerland sia or carcinoma in situ point to a higher risk of recurrence or progression [1,3]. Occasionally, reddish and mossy spots of the bladder mucosa allow the localization by biopsy of these foci of dysplasia, but mostly no specific changes of the bladder wall can be recognized by white light cystoscopy [4].

Although its accuracy is highly variable, urinary bladder cytology as a routine test in the early detection of bladder cancer and in the patient monitoring after treatment is well established, but does not enable concomitant urothelial atypia at the time of the first bladder tumor resection to be discovered. Moreover, the usefulness of random biopsies from normal-looking mucosa has recently been seriously questioned [5]. Therefore, as for epithelial cancers of other organs such as the oesophagus or the uterine cervix, there is a need to localize urothelial atypia during the endoscopic exploration.

Simple methods such as mucosal staining with methylene blue have failed due to lack of sensitivity and specificity [6]. Fluorescence studies correlated with histology on cystectomy specimens after intravenous injection of hematoporphyrin derivatives have demonstrated the possible role of a photodynamic method for detecting and localizing dysplasia and carcinoma in situ in bladder cancer [7]. However, the sophisticated equipment needed to obtain good images, the lack of selectivity of the photosensitizer and the risk of skin photosensitization have limited its application for clinical routine practice in urology. Recently, the high fluorescence contrast and intensity observed when treating cutaneous in situ tumors by photodynamic therapy using the endogenous photosensitizer protoporphyrin IX (PpIX), induced by the topical application of 5-aminolevulinic acid (ALA), has suggested the same approach to be suitable for detection and treatment of epithelial tumors of other organs, such as the oesophagus and the bladder [8]. The first clinical reports on the photodetection of early transitional cell carcinoma by light-induced fluorescence of PpIX following topical administration of ALA $[9,11]$ stimulated interest in this new method of mapping the bladder mucosa in search of dysplasia or carcinoma in situ. 
By comparison to the first system of bladder wall illumination with a krypton ion laser, the new light source, a xenon arc lamp, considerably simplifies the procedure by enabling the investigator to examine the bladder wall in real time without the necessity for an auxiliary device. This study presents our experience with this new system and attempts to assess the accuracy of the method in the detection of high-grade dysplasia and carcinoma in situ.

\section{Material and method}

Fifty milliliters of a $3 \%$ solution of ALA, buffered with $5 \mathrm{ml}$ phosphate-buffered saline (PBS) and adjusted to a $\mathrm{pH}$ of 5.3 with $7 \mathrm{ml}$ sodium hydroxide $(1 N)$, were instilled into the bladder through a 16 -French Foley catheter about $6 \mathrm{~h}$ before photodetection. Patients were asked to keep the catheter closed for $4 \mathrm{~h}$ to optimize the exposure of the bladder wall to the photosensitizing solution. The light source was a filtered xenon arc lamp which produced either white light for conventional cystoscopy or blue light with a wavelength range of $380-450 \mathrm{~nm}$ to excite the sensitized bladder mucosa. A foot pedal was used to switch from white to blue light and vice versa when necessary. The power of the fluorescence excitation light at the end of the cystoscope ranged between 150 and $480 \mathrm{~mW}$, giving under normal conditions of use a power density of $65 \mathrm{~mW} / \mathrm{cm}^{2}$. The white light, the blue excitation light and the resulting tissue fluorescence were transmitted through the optical fiber bundle of a Storz cystoscope or resectoscope. Two optics of $0^{\circ}$ and of $30^{\circ}$, suitable for either a cystoscope or resectoscope sheath, were specially configured to optimize the light transmission. As the PpIX emission peak was $635 \mathrm{~nm}$, a long-pass filter $(\lambda>490 \mathrm{~nm})$ was adapted to the ocular piece of the cystoscope to transmit the red and cut out most of the blue-reflected excitation light. Thus, the fluorescent spots appeared red, whereas the non-fluorescent mucosa stayed green. The somewhat darker bladder wall vessels facilitated the positioning of the cytoscope during the operation.

Starting in January 1995, 33 bladder examinations were performed in 31 patients, 23 male patients and 8 female patients (two patients had two successive operations). Mean age was 66.1, ranging from 44 to 84 years. Eleven patients were operated on for the first time, 6 for the second time and 16 for the third time or more. Topical chemotherapy or immunotherapy with bacille Calmette-Guérin (BCG) was added to the previous surgical treatment in 19 patients. Bladder urinary cytological examination was regularly performed when the catheter was introduced for the ALA instillation.

The operation always started with a careful bladder inspection under white light in order to note the presence, number and location of all the visible tumors and suspicious areas. To evaluate the sensitivity and the specificity of the method in patients with bladder tumors, a four-quadrant random biopsy of the normal bladder mucosa was performed for each patient as follows: the randomly designated spot of normal mucosa under white light was excited under blue light to determine the fluorescence response and then immediately sampled by forceps for biopsy. A total of 132 random biopsies were obtained in this way, 51 in fluorescent areas and 81 in nonfluorescent areas. All other fluorescent areas on an apparently normal or slightly irregular mucosa under white light were also biopsied and sent for analysis. The transurethral resection of significant tumors was generally performed under fluorescence, except in the case of major bleeding. Papillary and nonpapillary urothelial carcinomas were graded and staged according to the WHO 1973 classified [12] and the IUCC/AJC-1992 system [13], respectively. Flat intraepithelial neoplastic lesions were graded according to the criteria of Nagy et al. [14], and classified as grade 1 (mild dysplasia), grade 2 (moderate dysplasia), grade 3 (marked dysplasia) and in situ carcinoma.

\section{Results}

No systemic or local side effects following ALA bladder instillation either before or after the photodetection procedure were noted. Except for one pTaG1 tumor, all papillary tumors visible on white light cystoscopy were fluorescent. The correlations between the fluorescence findings and the presence or absence of cancer cells for the 132 random biopsies on apparently normal mucosa are shown in Table 1. The predictive value of a positive fluorescence corresponding to a tumor is estimated at $66.6 \%$ (34/51), and the predictive value of a negative fluorescence corresponding to benign tissue at $91.3 \%(74 / 81)$. The sensitivity of this photodetection method in superficial bladder cancer can be estimated at $82.9 \%(34 / 41)$ and the specificity at $81.3 \%(74 / 91)$.

In $22 / 33$ patients, $66.6 \%$ of our collective, 47 tumors were detected by the fluorescence (Table 2). Seven of these 22 patients (31.8\%) had grade 3 dysplasia or carcinoma in situ (CIS). Table 3 shows the correlations between cytology, white light cystoscopy and fluorescence findings for each patient. The fluorescence method detected additional urothelial atypia, invisible under white light, in $12 / 14$ patients $(85 \%)$ with multiple tumors and in $5 / 12$ patients $(41 \%)$ with a single tumor. In contrast to the fluorescence cytoscopy, urinary cytology was not really helpful in patients with macroscopic tumors. For patients previously operated on for a superficial bladder cancer, who presented no specific lesion on white light cystoscopy, fluorescence cystoscopy confirmed the absence of neoplastic lesions in three and detected urothelial atypia or even a small tumor in four of them. In these cases, urinary cytology could only give an indication of the presence or absence of cancerous cells, whereas fluorescence cytoscopy precisely delineated the number of carcinomatous spots.

Table 1 Correlation between fluorescence findings and histopathological diagnosis for the 132 random biopsies on apparently normal mucosa in superficial bladder cancer

\begin{tabular}{lllr}
\hline & $\begin{array}{l}\text { Positive } \\
\text { histology }\end{array}$ & $\begin{array}{l}\text { Negative } \\
\text { histology }\end{array}$ & Total \\
\hline Positive fluorescence & 34 & 17 & 51 \\
Negative fluorescence & 7 & 74 & 81 \\
& 41 & 91 & 132 \\
\hline
\end{tabular}

Table 2 Diagnosis distribution of the 47 tumors invisible by white light cystoscopy and detected by fluorescence in $22 / 33$ patients

\begin{tabular}{lrll}
\hline "Flat" intraepithelial tumors & 41 & Papillary tumors & 6 \\
\hline Grade 1 dysplasia & 12 & pTaG1 & 2 \\
Grade 2 dysplasia & 15 & pTaG2 & 3 \\
Grade 3 dysplasia & 3 & pT1G3 & 1 \\
Carcinoma in situ & 11 & & \\
\hline
\end{tabular}


Table 3 Correlation between cytology, white light cystoscopy (CUS) and fluorescence findings

\begin{tabular}{|c|c|c|c|c|c|c|c|c|c|}
\hline \multirow[t]{2}{*}{ Cytology } & \multirow[t]{2}{*}{ White light CUS ${ }^{a}$} & & \multicolumn{4}{|c|}{ Diagnosis } & \multicolumn{3}{|c|}{ Fluorescence $\mathrm{CUS}^{\mathrm{b}}$} \\
\hline & & & TaG1 & $\mathrm{TaG} 2$ & $\mathrm{~T} 1 \mathrm{G} 2$ & $\mathrm{~T} 1 \mathrm{G} 3$ & Dys G1-2 & Dys G3-CIS & $\mathrm{TaG} 2$ \\
\hline Correct $6 x$ & Multiple tumors & 14 & 3 & 7 & 1 & 3 & 9 & 3 & 0 \\
\hline Correct $5 \times$ & Unique tumor & 12 & 6 & 5 & 0 & 1 & 3 & 2 & 0 \\
\hline Correct $5 x$ & No specific lesion & 7 & & \multicolumn{3}{|c|}{ No tumor } & 1 & 1 & 2 \\
\hline
\end{tabular}

a Except one pTaG1 tumor, all papillary tumors, visible by white light cystoscopy, were fluorescent

b Additional tumors invisible under white light and detected by fluorescence

\section{Discussion}

In the Canton of Vaud in Switzerland, the incidence of superficial bladder cancer is around 18.6/100000 per year in male patients and $4.2 / 100000$ per year in female patients, which represents a ratio of $4: 1$ [15]. The individual risk of cancer progression, reflecting the natural history of the disease, varies from 4 to $45 \%$ [16]. Moreover, in the year following the first treatment, superficial bladder cancer tends to recur in a proportion up to $70 \%$; and $10-20 \%$ of recurrences on the site of the initial tumor grow from carcinomatous foci left during the first operation [17]. If the characterization of dysplastic changes in the urothelium is important in the evaluation of the disease prognosis and the risk of recurrence, the surgical treatment of all exophytic and "flat" tumors during the first operation is mandatory.

So far no reliable method has been available to determine the cancer cells in delineating the tumor basis and screening urothelial atypia. PpIX-induced fluorescence detection of urothelial cancer cells by topical administration of ALA is a simple and harmless method for this. No local or systemic reaction was noted after ALA bladder instillation or illumination with the xenon blue light. The correlation between the fluorescence tissue response and the histopathological diagnosis on normal mucosa in superficial bladder cancer establishes that a fluorescent spot corresponds to a carcinoma in about two out of three cases and above all that a nonfluorescent spot corresponds to benign tissue in nine out of ten cases. Undoubtedly, fluorescence cystoscopy aids in the biopsy of the urothelium at the right place in bladder cancer. The electrocoagulation of false-positive fluorescent areas, due to inflammatory or hyperplastic cells, does not harm the bladder with normal compliance.

The sensitivity and the specificity of the procedure on apparently normal mucosa in patients with superficial bladder cancer is estimated at $82.9 \%$ and $81.3 \%$, respectively. Calculated on a per patient basis it has been estimated at $94.2 \%$ by the Munich group [10]. Following the same approach in our collective, bladder biopsies revealed dyspastic urothelial changes or CIS in 23 patients, among whom 22 had a positive fluorescence. Thus, our per patient estimated sensitivity is near $95.6 \%$, an equivalent value to the previous estimation.
Fluorescence cystoscopy constitutes a previous aid to white light cystoscopy. It is a simple and a reliable method in mapping the mucosa in bladder cancer. It facilitates the delineation of tumors and the screening of occult dysplastic changes, especially in multiple papillary bladder disease. In addition, it appears very useful in monitoring the patients after treatment.

Subsequent studies will be needed to assess whether the optimal transurethal bladder resection obtained with fluorescence cytoscopy contributes to a reduction of the risk of recurrence in superficial bladder cancer.

Acknowledgement This study has been supported by the Vaud-Geneva Foundation.

\section{References}

1. Olsen PR, Wolf H, Schroeder T, Fischer A, Hojgaard K (1988) Urothelial atypia and survival rate of 500 unselected patients with primary transitional-cell tumour of the urinary bladder. Scand J Urol Nephrol 22:257

2. Witjes JA, Kiemeney LALM, Oosterhof GON, Debruyne FMJ (1992) Prognostic factors in superficial bladder cancer. Eur Urol 21:89

3. Wolf H, Hojgaard K (1983) Urothelial dysplasia concomitant with bladder tumours as a determinant factor for future new occurrences. Lancet 8340:134

4. Wallace DMA, Hindmarsh JR, Webb JN, Busuttil A, Hardgreave TB, Newsam JE, Chisholm GD (1979) The role of multiple mucosal biopsies in the management of patients with bladder cancer. Br J Urol 51:535

5. Kiemeney LALM, Witjes JA, Heijbroek RP, Koper NP, Verbeek ALM, Debruyne FMJ and the Members of the Dutch South-East Co-operative Urological Group (1994) Should random urothelial biopsies be taken from patients with primary superficial bladder cancer? A decision analysis. Br J Urol 73:164

6. Vicente J, Chéchile G, Algaba F (1987) Value of in vivo mucosa-staining test with methylene blue in the diagnosis of pretumoral and tumoral lesions of the bladder. Eur Urol 13:15

7. Benson RC, Farrow GM, Kinsey JH, Cortese DA, Zincke H, Utz DC (1982) Detection and localization of in situ carcinoma of the bladder with hematoporphyrin derivative. Mayo Clin Proc $57: 548$

8. Kennedy JC, Pottier RH, Pross DC (1990) Photodynamic therapy with endogenous protoporphyrin IX: Basic principles and present clinical experience. J Photochem Photobiol B: Biol $6: 143$

9. Kriegmair M, Baumgartner R, Knuechel R, Steinbach P, Ehsan A, Lumper W, Hofstädter F, Hofstetter A (1994) Fluorescence photodetection of neoplastic urothelial lesions following intravesical instillation of 5-aminolevulinic acid. Urology $44: 836$ 
10. Kriegmair M, Baumgartner R, Knuechel R, Stepp H, Hofstädter F, Hofstetter A (1996) Detection of early bladder cancer by 5 -aminolevulinic acid induced porphyrin fluorescence. J Urol 155:105

11. Jichlinski P, Forrer M, Mizeret J, Glanzmann T, Braichotte D, Wagnières G, Zimmer G, Guillou L, Schmidlin F, Graber P, van den Bergh $\mathrm{H}$, Leisinger $\mathrm{HJ}$ (in press) Clinical evaluation of a method for detecting superficial transitional cell carcinoma of the bladder by light induced fluorescence of protoporphyrin IX following topical application of 5-aminolevulinic acid. Preliminary results. Lasers Surg Med (in press)

12. Mostofi FK, Sobin LH, Torloni H (1973) Histological typing of urinary bladder tumours. In: World Health Organisation (ed) International histological classification of tumours. WHO, Geneva

13. UICC (1992) TNM classification of malignant tumours, 4th edn. Springer-Verlag, Berlin Heidelberg New York
14. Nagy GK, Frable WJ, Murphy WM (1982) Classification of premalignant urothelial abnormalities. A Delphi study of the $\mathrm{Na}-$ tional Bladder Cancer Collaborative Group A. In: Sommers SC, Rosen PP (eds) Pathology Annual, Appleton-Century-Crofts, Norwalk, p 219

15. Levi F, La Vecchia C, Randimbison L, Franceschi S (1993) Incidence of infiltrating cancer following superficial bladder carcinoma. Int J Cancer 55:419

16. Abel PD (1993) Follow-up of patients with "superficial" transitional cell carcinoma of the bladder: the case for a change in policy. Br J Urol 72:135

17. Klän R, Loy V, Huland H (1991) Residual tumor discovered in routine second transurethral resection in patients with stage T1 transitional cell carcinoma of the bladder. J Urol 146:316 